\section{Commentary: Gait speed in aortic surgery - do we need a pathway for slow walkers?}

\author{
Dinela Rushani, MD, ${ }^{\mathrm{a}}$ Rachel Eikelboom, MD, ${ }^{\mathrm{b}, \mathrm{c}}$ \\ Rakesh C. Arora, MD, PhD, , c and \\ Bobby Yanagawa, MD, PhD, FRCSC ${ }^{a}$
}

In this issue of The Journal of Thoracic and Cardiovascular Surgery, Hobbs et al ${ }^{1}$ retrospectively reviewed 453 patients aged $\geq 60$ years undergoing elective aortic surgery whose 5-meter gait speed was recorded in the Society for Thoracic Surgery database. They found that $30 \%$ of patients had a slow gait speed $(\leq 0.83 \mathrm{~m} / \mathrm{s})$ and, not surprisingly, "slow walkers" were older with more comorbidities. A slow 5-meter gait speed was predictive of a worse adjusted composite outcome of renal failure, prolonged mechanical ventilation, discharge to a location other than home, and in-hospital mortality (odds ratio, 2.08; 95\% confidence interval, .27-3.40; $P=.004) .{ }^{1}$ The observation was that a $1-\mathrm{m} / \mathrm{s}$ increase in gait speed was associated with a $73 \%$ reduction in the risk of the composite primary outcome. As a caveat, the composite endpoint included a range of outcomes, and the difference was driven by outcomes other than mortality (ie, discharge location). ${ }^{1}$ Other patientcentered outcomes relevant to frail patients, such as functional status, functional recovery, and health-related quality of life, were not captured. As a frailty tool, the 5-meter gait speed test is 1) simple; 2) provides additional predictive value to traditional risk predictors; and 3 ) conveys actionable information by identifying opportunities to modify risk or informing decision-making about

\footnotetext{
From the ${ }^{\mathrm{a} D i v i s i o n}$ of Cardiac Surgery, St. Michael's Hospital, University of Toronto, Toronto, Ontario, Canada; ${ }^{\mathrm{b}}$ Department of Surgery, Max Rady College of Medicine, Winnipeg, Manitoba, Canada; and ${ }^{\mathrm{c} C a r d i a c}$ Sciences Program, St. Boniface Hospital, Winnipeg, Manitoba, Canada.

Disclosures: R.C.A. has received an unrestricted educational grant from Pfizer Canada, Inc., and honoraria from Mallinckrodt Pharmaceuticals, Abbott Nutrition, and Edwards LifeSciences for work unrelated to the present report.

Received for publication March 31, 2020; revisions received March 31, 2020; accepted for publication April 1, 2020; available ahead of print April 9, 2020.

Address for reprints: Bobby Yanagawa, MD, PhD, FRCSC, Division of Cardiac Surgery, St. Michael's Hospital, University of Toronto, 30 Bond St, 8th Floor, Bond Wing, Toronto, Ontario M5B 1W8, Canada (E-mail: yanagawab@smh.ca). J Thorac Cardiovasc Surg 2022;163:895-6 $0022-5223 / \$ 36.00$

Copyright (c) 2020 by The American Association for Thoracic Surgery https://doi.org/10.1016/j.jtcvs.2020.04.001
}

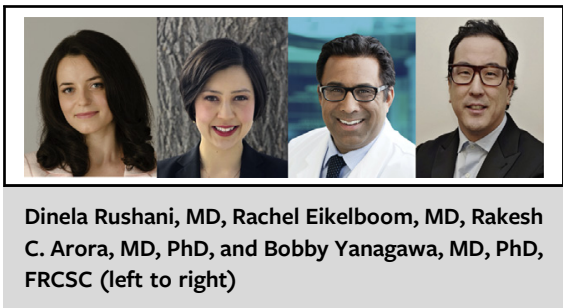

CENTRAL MESSAGE

The 5-meter gait speed can predict adverse outcomes for patients undergoing elective aortic surgery and thus could help to identify high-risk patients for targeted interventions.

surgical intervention. ${ }^{2,3}$ Furthermore, frailty carries substantial health and economic effects for patients undergoing cardiac surgery. ${ }^{4}$ Thus, we congratulate the authors on this important study, which has added to the increasing data demonstrating the 5-meter gait speed test as an important adjunct to traditional risk calculators, such as the Society for Thoracic Surgery score and the EuroSCORE. ${ }^{5,6}$

Given that frailty is a risk factor for mortality after coronary artery bypass grafting and valve surgery, it should not come as a surprise that it is also a risk factor for adverse events after aortic surgery. ${ }^{2}$ However, for coronary disease and valvulopathy, established and emerging percutaneous alternatives to open surgery are available. As such, frailty measures could significantly alter the treatment path. For patients requiring proximal aortic surgery, with few exceptions, open surgery with sternotomy is the only definitive treatment (although experimental procedures such as Endo-Bentall could be on the horizon). So, how can a frailty assessment positively affect patient treatment?

We propose that frail patients could be optimally treated with a "frailty enhanced recovery protocol" (ERP). Such an ERP could involve preoperative coordinated multidisciplinary care that includes geriatric specialists, nursing care, nutritionists, pharmacists, physical therapy, occupational therapy, social work, and others. This would seek methods for preoperative risk factor modification or "prehabilitation" and older adult-focused perioperative care that incorporate strategies to reduce the risk of catheter-based urinary tract infection, pressure ulcers, falls, and delirium. Alvarez-Nebreda et $\mathrm{al}^{7}$ reported one such 
perioperative frailty pathway on behalf of the Society for Perioperative Assessment and Quality Improvement. They focused on prehabilitation, including medication optimization, psychological support, physical exercise, and nutritional optimization, as well as perioperative geriatric co-management in a multidisciplinary team with standardized protocols, early mobilization, and advance discharge planning to avoid hospital readmission.

In our morbidity and mortality rounds, the root cause analysis of major adverse events often points to frailty as a major contributing factor resulting in failure-to-rescue (ie, death after an unanticipated complication). The 5-meter gait speed test could be one simple and practical screening test for frailty in our surgical patients. If we mobilize our valuable resources under the guidance of our geriatricians, we might be able to place such patients on an ERP or pathway to limit such adverse events.

\section{References}

1. Hobbs RD, Norton EL, Wu X, Willer CJ, Hummell SL, Prager RL, et al. Gait speed is a preoperative indicator of postoperative events after elective proximal aortic surgery. J Thorac Cardiovasc Surg. 2022;163:886-94.e1.

2. Yanagawa B, Graham MM, Afilalo J, Hassan A, Arora RC. Frailty as a risk predictor in cardiac surgery: beyond the eyeball test. J Thorac Cardiovasc Surg. 2018; 156:172-6.e2.

3. Hogan DB, Maxwell CJ, Afilalo J, Arora RC, Bagshaw SM, Basran J, et al. A Scoping review of frailty and acute care in middle-aged and older individuals with recommendations for future research. Can Geriatr J. 2017;20:22-37.

4. Yanagawa B, Latter DA, Fedak PWM, Cutrara C, Verma S. The cost of frailty in cardiac surgery. Can J Cardiol. 2017;33:959-60.

5. Afilalo J, Eisenberg MJ, Morin JF, Bergman H, Monette J, Noiseux N, et al. Gait speed as an incremental predictor of mortality and major morbidity in elderly patients undergoing cardiac surgery. J Am Coll Cardiol. 2010;56:1668-76.

6. Afilalo J, Kim S, O'Brien S, Brennan JM, Edwards FH, Mack MJ, et al. Gait speed and operative mortality in older adults following cardiac surgery. JAMA Cardiol. 2016;1:314-21.

7. Alvarez-Nebreda ML, Bentov N, Urman RD, Setia S, Huang JC, Pfeifer K, et al. Recommendations for preoperative management of frailty from the Society for Perioperative Assessment and Quality Improvement (SPAQI). J Clin Anesth. 2018;47:33-42.
See Article page 886.

\section{Commentary: Something in the way they move: Does gait speed predict postoperative events following elective proximal aortic surgery?}

\author{
David C. Liu, MD, and Kamal R. Khabbaz, MD
}

Surgeons have long known of intangibles that give pause to recommending a patient for cardiac surgery out of concern for increased risk. These intangibles were ascribed to the art of medicine, the so-called eyeball test. Evidence-based

\footnotetext{
From the Division of Cardiac Surgery, Department of Surgery, Beth Israel Deaconess Medical Center and Harvard Medical School, Boston, Mass.

Disclosures: The authors reported no conflicts of interest.

The Journal policy requires editors and reviewers to disclose conflicts of interest and to decline handling or reviewing manuscripts for which they may have a conflict of interest. The editors and reviewers of this article have no conflicts of interest.

Received for publication April 9, 2020; accepted for publication April 11, 2020; available ahead of print April 23, 2020.

Address for reprints: Kamal R. Khabbaz, MD, Division of Cardiac Surgery, Department of Surgery, Beth Israel Deaconess Medical Center, 110 Francis St, Suite 2A, Boston, MA 02215 (E-mail: kkhabbaz@bidmc.harvard.edu).

J Thorac Cardiovasc Surg 2022;163:896-7

$0022-5223 / \$ 36.00$

Copyright (c) 2020 by The American Association for Thoracic Surgery

https://doi.org/10.1016/j.jtcvs.2020.04.046
}

Check for updates

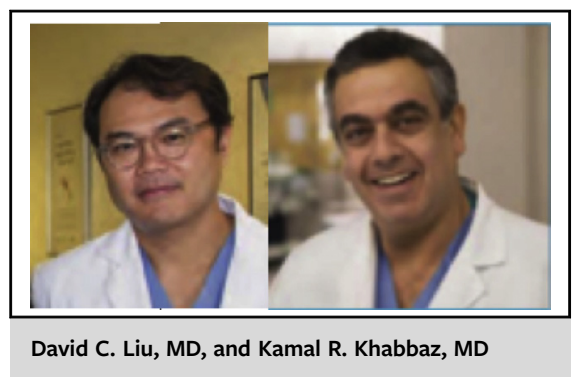

CENTRAL MESSAGE

Slow gait speed, a marker of

frailty, is a risk factor for post-

operative events after elective

proximal aortic surgery.

medicine has given a face to these intangibles we now define as frailty. Many studies have shown frailty to be a significant predictor of postoperative mortality and morbidity after cardiac surgery, including coronary surgery, valve surgery, and transcatheter aortic valve replacement. ${ }^{1-3}$ Gait speed is a recognized surrogate for frailty. ${ }^{4}$ It is a simple test that is easy to administer.

Hobbs and colleagues ${ }^{5}$ used the preoperative 5-m gait speed test to determine whether it is a predictor of adverse postoperative events in patients undergoing proximal aortic 